\title{
ZERO-EXTREME POINTS AND THE GENERALIZED CONVEX KERNEL
}

\author{
ARTHUR G. SPARKS
}

\begin{abstract}
Let $S$ be a compact simply connected set in the plane. Let $K(n)$ denote the generalized convex kernel of $S$ of order $n$, bd $S$ the boundary of $S, E(0, S)$ the set of 0 -extreme points of $S$, and for $x \in S$, let $K(n, x)$ denote the $n$th order convex kernel of $x$ in $S$. It is known that $K(n)=$ $\bigcap\{K(n, x) \mid x \in$ bd $S\}$ and in certain cases, it is known that $K(1)=$ $\bigcap\{K(1, x) \mid x \in E(0, S)\}$. The main result of this paper extends the above mentioned results for certain sets. It is shown that $K(n)=\bigcap\{K(n, x) \mid x \in$ $E(0, S)\}$ for certain compact simply connected sets $S$ in the plane. In the process of obtaining this result, a characterization of the boundary is also obtained.
\end{abstract}

1. Introduction. Let $S$ be a compact set in $E_{2}$. A point $p$ in $S$ is called a 0 -extreme point of $S$ if and only if it is not interior to a segment in $S$. The set of 0 -extreme points of $S$ will be denoted by $E(0, S)$. A point $q$ in $S$ is called an extreme point of $S$ if and only if it is not interior to a segment with endpoints in $S$. For $x$ in $S$, let $K(n, x)$ denote the set of points that can be joined to $x$ by some polygonal $k$-path in $S$, where $k \leqslant n$. The generalized convex kernel $K(n)$ is defined by $K(n)=\{x \mid S \subset K(n, x)\}$.

In a paper by Kenelly, Hare, Evans and Ludescher [2], a result was proved which in $E_{2}$ is as follows: let $S$ be a compact set in $E_{2}$ such that $K(1) \neq \varnothing$, then $K(1)=\bigcap\{K(1, x) \mid x \in E(0, S)\}$.

In a paper by Bruckner and Bruckner [1], the following was proved: let $S$ be a compact simply-connected set in $E_{2}$, then $K(n)=\bigcap\{K(n, x) \mid x \in$ bd $S$ \}.

The main concern of this paper is to sharpen the second result and to provide a generalization of the first (for certain sets). It will be proved that if $S$ is a compact Jordan set in $E_{2}$, then $K(n)=\bigcap\{K(n, x) \mid x \in E(0, S)\}$.

There are numerous examples to show that this result cannot be refined further by taking the intersection over the set of extreme points of $S$. One such example follows.

Example. Let $J$ be the closed polygonal path whose vertices (in order) are as follows: $(-3,-3),(-3,3),(3,3),(3,-3),(1,-3),(2,0),(-2,0)$ and

Presented to the Society, August 27, 1970 under the title On zero-extreme points and the generalized convex kernel; received by the editors September 3, 1974 and, in revised form, July 10, 1975 and April 25, 1977.

AMS (MOS) subject classifications (1970). Primary 52-XX, 52A10.

Key words and phrases. Zero-extreme points, extreme points, convex kernel, generalized convex kernel.

(C) American Mathematical Society 1977 
$(-1,-3)$. Then $J$ is a closed Jordan curve. Let $S$ be the set consisting of $J$ along with its interior, then $S$ is a compact simply-connected set. Label the vertices $x_{1}$ through $x_{8}$, successively. Then the set of extreme points of $S$ is $\operatorname{Ext}(S)=\left\{x_{1}, x_{2}, x_{3}, x_{4}\right\}$. Let $y=(-3,-1)$, then $y \in \cap\{K(2, x) \mid x \in$ $\operatorname{Ext}(S)\}$. However, $y \notin K(2)$ since $y \notin K\left(2, x_{5}\right)$.

2. Preliminaries. Throughout this paper, all sets will be in $E_{2}$. If $A$ is a set, then $\bar{A}$ will denote its closure, bd $A$ its boundary, $A^{c}$ its complement, and int $A$ its interior. If $B$ is a set, then $A-B$ will denote the complement of $B$ relative to $A$. For points $p$ and $q,[p, q]$ will denote the closed segment with endpoints $p$ and $q,(p, q)$ the corresponding open segment, and $L(p, q)$ the line determined by $p$ and $q$.

Definition. A compact set $S$ is said to be simply-connected if and only if $S^{c}$ is connected.

It is most important to keep in mind that if $J$ is a closed Jordan curve in the simply-connected set $S$, then the interior of $J$ is contained in $S$, where the interior of $J$ is in the sense of the Jordan Curve Theorem.

Definition. The set $S$ is said to be an $L_{n}$ set if $K(n, x)=S$ for every $x \in S$; i.e., $K(n)=S$.

\section{Properties of the boundary.}

DEFINITION. A domain is an open connected set. A domain whose boundary is a simple closed curve is called a Jordan domain. A compact set which is the closure of a Jordan domain is called a compact Jordan set.

Definition. Let $D$ be a domain. A cross-cut of $D$ is a simple arc whose endpoints are in bd $D$ and all its other points are in $D$.

TheOREM 1. Let $S$ be a compact Jordan set with boundary $J$. If $z$ is not a point of local convexity of $S^{c}$, then $z \in \overline{E(0, S)}$.

Proof. Let $S$ be a compact Jordan set with boundary $J$ and interior $D$. Suppose $z \in J$ and $z$ is not a point of local convexity of $S^{c}$.

Let $\varepsilon>0$ and let $\varepsilon^{*}$ satisfy the inequality $0<\varepsilon^{*}<\varepsilon$. Then the $\varepsilon^{*}$-neighborhood of $z, N\left(\varepsilon^{*} ; z\right)$, has the property that $N\left(\varepsilon^{*} ; z\right) \cap S^{c}$ is not convex. Thus, there exist $p_{1}, p_{2} \in N\left(\varepsilon^{*} ; z\right) \cap S^{c}$ such that $\left[p_{1}, p_{2}\right] \not \subset N\left(\varepsilon^{*} ; z\right) \cap S^{c}$. Hence, it follows that $\left[p_{1}, p_{2}\right] \not \subset S^{c}$ and thus $\left[p_{1}, p_{2}\right] \cap S \neq \varnothing$. Since $J$ is a Jordan curve and $S^{c}$ is open, we know that $p_{1}$ and $p_{2}$ can be chosen to have the additional properties that $\left[p_{1}, p_{2}\right]$ contains a cross-cut $\left[q_{1}, q_{2}\right]$ of $D$ and that one of the two components of $D-\left[q_{1}, q_{2}\right]$ is a subset of $N\left(\varepsilon^{*} ; z\right)$. Denote this component by $D_{1}$ and let $L_{1}$ be the subarc of $J$ such that bd $D_{1}=\left[q_{1}, q_{2}\right] \cup L_{1}$. (See Figures 1 and 2.)

Let $L\left(q_{1}, q_{2}\right)$ denote the line determined by $q_{1}$ and $q_{2}$. Now $D_{1}$ is an open, nonempty set and $\bar{D}_{1}$ is compact. Hence, there exists a line $H$ such that:

1. $H$ is parallel to $L\left(q_{1}, q_{2}\right)$ but $H \neq L\left(q_{1}, q_{2}\right)$;

2. $H \cap L_{1} \neq \varnothing$;

3. $H \cap \overline{D_{1}}=H \cap L_{1}$. 


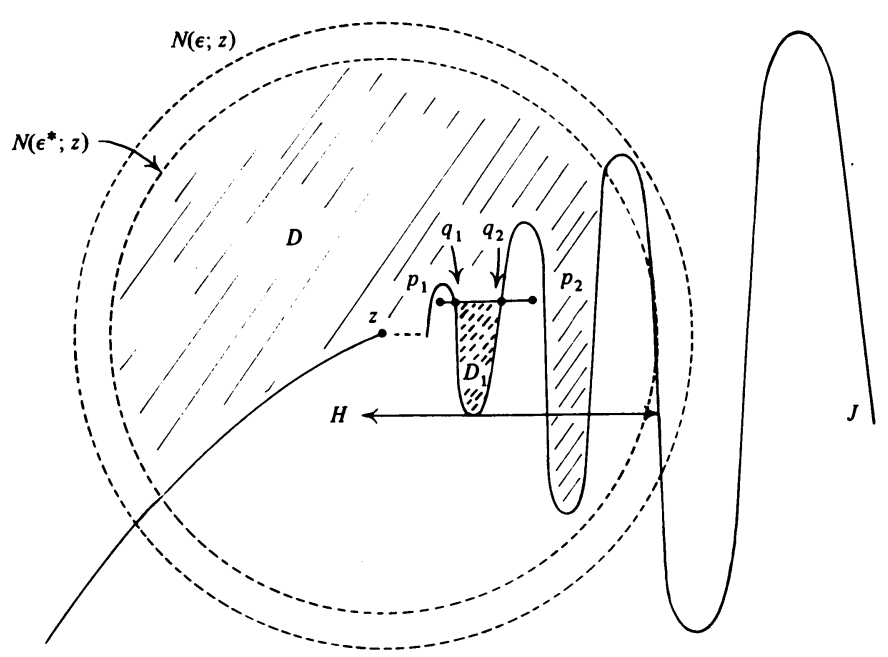

Figure 1

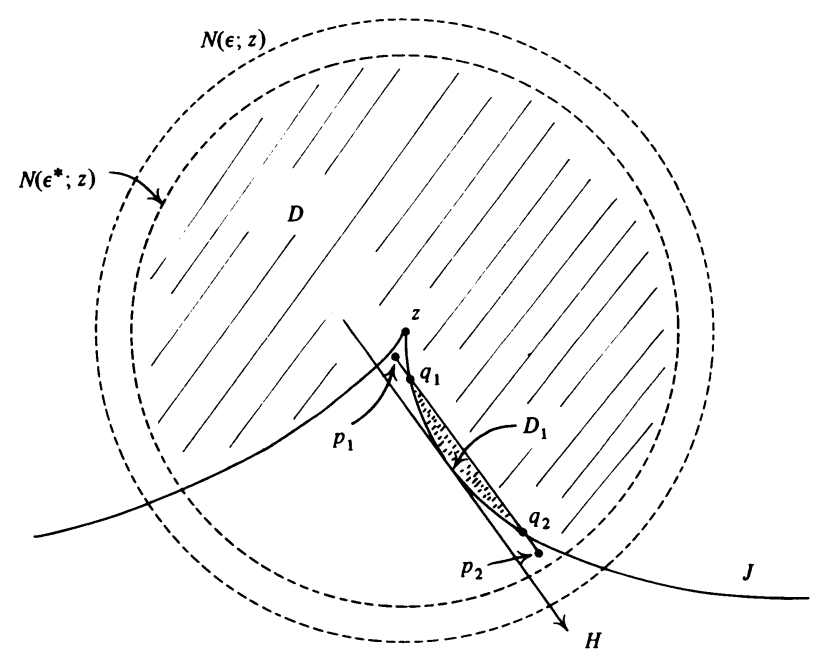

Figure 2

Since $\overline{D_{1}}$ is compact and $H$ is closed, it follows that $H \cap \overline{D_{1}}$ is compact. Hence, $H \cap L_{1}$ is compact. Since $H \cap L_{1}$ is a nonempty compact subset of the line $H$, its components consist of closed segments and/or singleton sets. If $\{y\}$ is a component of $H \cap L_{1}$, then clearly $y \in E(0, S)$. If $\left[y_{1}, y_{2}\right]$ is a component of $H \cap L_{1}$, then clearly $y_{1}, y_{2} \in E(0, S)$. Thus, $\left(H \cap L_{1}\right) \cap$ $E(0, S) \neq \varnothing$.

Now, $D_{1} \subset N\left(\varepsilon^{*} ; z\right)$ implies that $\overline{D_{1}} \subset N(\varepsilon ; z)$. Thus $\left(H \cap \overline{D_{1}}\right) \subset N(\varepsilon ; z)$ and hence $\left(H \cap L_{1}\right) \subset N(\varepsilon ; z)$. It now follows that $N(\varepsilon ; z) \cap E(0, S) \neq \varnothing$. Since $\varepsilon>0$ was arbitrary, it follows that $z \in \overline{E(0, S)}$.

Defintion. Let $S$ be a compact Jordan set. A Jordan arc $L$ is said to be a concave arc of $S$ if:

(i) $L \subset$ bd $S$, 
(ii) the endpoints of $L$ are in $\overline{E(0, S)}$, and

(iii) if $x \in L$ and $x$ is not an endpoint of $L$, then $x$ is a point of local convexity of $S^{c}$.

THEOREM 2. Let $S$ be a compact Jordan set and let $\Phi(S)=[\overline{E(0, S)}]^{c}$ $\cap$ bd $S$. Let $L$ be an arc with endpoints $a$ and $b$. Then $L$ is a concave arc of $S$ if and only if $L-\{a, b\}$ is a component of $\Phi(S)$.

Proof. Suppose that $L-\{a, b\}$ is a component of $\Phi(S)$ and let $x \in L-$ $\{a, b\}$.

If $x$ is not a point of local convexity of $S^{c}$, then $x \in \overline{E(0, S)}$ by Theorem 1. However, this is impossible by the definition of $\Phi(S)$. Thus it follows that $x$ is a point of local convexity of $S^{c}$. Hence, $L$ is a concave arc of $S$.

Now suppose that $L$ is a concave arc of $S$.

If $x \in L-\{a, b\}$, it is easily seen that $x \notin E(0, S)$. It follows that $L-\{a, b\}$ is a component of $\Phi(S)$.

THEOREM 3. Let $S$ be a compact Jordan set and let $L$ be a concave arc of $S$ with endpoints $a$ and $b$. If $x \in S$ is such that $a, b \in K(n, x)$, then $L \subset$ $K(n, x)$.

Proof. Suppose $P_{n}(x, a)$ and $P_{n}(x, b)$ are $n$-paths in $S$ joining $x$ to $a$ and $b$ respectively. Letting $P_{n}=P_{n}(x, a) \cup P_{n}(x, b)$, it is clear that $P_{n} \subset K(n, x)$.

Let $y \in L-\{a, b\}$, then $y \in$ bd $S$. Also, from Theorem 2, we know that $y \notin E(0, S)$. Thus, there is a segment $[p, q]$ such that $[p, q] \subset S$ and $y \in$ $(p, q)$. By the compactness of $S,[p, q]$ can be extended to a maximal segment $\left[p^{*}, q^{*}\right]$ in $S$ such that $[p, q] \subset\left[p^{*}, q^{*}\right]$. Since $L$ is a concave arc and $S$ is simply-connected, it follows that $P_{n} \cap\left[p^{*}, y\right] \neq \varnothing$ and $P_{n} \cap\left[y, q^{*}\right] \neq \varnothing$ (see Figure 3). (Recall that since $S$ is simply-connected, the points "enclosed

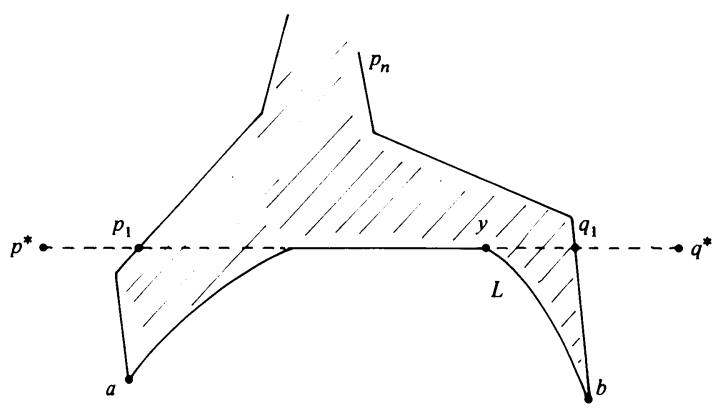

FIGURE 3

by $P_{n} \cup L^{\prime \prime}$ are also in $S$. Thus, if either $P_{n} \cap\left[p^{*}, y\right]$ or $P_{n} \cap\left[y, q^{*}\right]$ were empty, then $\left[p^{*}, q^{*}\right]$ could be extended, thus contradicting its maximality.) Letting $p_{1} \in P_{n} \cap\left[p^{*}, y\right]$ and $q_{1} \in P_{n} \cap\left[y, q^{*}\right]$, it follows that $p_{1}, q_{1} \in$ $K(n, x)$ and $y \in\left[p_{1}, q_{1}\right]$. From [4], we know that $\left[p_{1}, q_{1}\right] \subset K(n, x)$ and thus $y \in K(n, x)$. Since $y$ was arbitrary in $L-\{a, b\}$, it is clear that $L-\{a, b\}$ $\subset K(n, x)$. Since $a, b \in K(n, x)$, it now follows that $L \subset K(n, x)$. 
THEOREM 4. Let $S$ be a compact Jordan set. Then $K(n)=\bigcap\{K(n, x) \mid x \in$ $E(0, S)\}$.

Proof. Since $E(0, S) \subset$ bd $S$, it follows that

$$
\cap\{K(n, x) \mid x \in \text { bd } S\} \subset \cap\{K(n, x) \mid x \in E(0, S)\} .
$$

From [1], we know that $K(n)=\bigcap\{K(n, x) \mid x \in$ bd $S\}$. Thus, it is clear that $K(n) \subset \cap\{K(n, x) \mid x \in E(0, S)\}$. To prove the desired equality, we need only show the reverse containment.

Thus, suppose $z \in \cap\{K(n, x) \mid x \in E(0, S)\}$. Then $E(0, S) \subset K(n, z)$. From [1], we know that $K(n, z)$ is compact. Hence, $\overline{E(0, S)} \subset K(n, z)$. By Theorems 2 and 3 , it follows that $\Phi(S) \subset K(n, z)$. Since bd $S=\Phi(S) \cup \overline{E(0, S)}$, it is clear that bd $S \subset K(n, z)$. Hence, $z \in K(n, y)$ for every $y \in$ bd $S$ and therefore $z \in \cap\{K(n, y) \mid y \in$ bd $S\}$. Thus, $z \in$ $K(n)$. Hence, the reverse containment has been verified and thus the desired equality has been proved.

\section{REFERENCES}

1. A. M. Bruckner and J. B. Bruckner, Generalized convex kernels, Israel J. Math. 2 (1964), $27-32$.

2. J. W. Kenelly, W. R. Hare, Jr., B. D. Evans and W. H. Ludescher, Convex components, extreme points, and the convex kernel, Proc. Amer. Math. Soc. 21 (1969), 83-8\%.

3. M. H. A. Newman, Elements of the topology of plane sets of points, Cambridge Univ. Press, London and New York, 1961, pp. 115-119.

4. A. G. Sparks, Intersections of maximal $L_{n}$ sets, Proc. Amer. Math. Soc. 24 (1970), 245-250.

Department of Mathematics, Georgia Southern College, Statesboro, Georgia 30458 